\title{
Andioxidant and structural analysis of polysaccharide extracted from Clitocybe maxima Stipe using ultrasonication: simulation and validation
}

\section{K. Thirugnanasambandham}

Department of Chemistry, ECET-638052, Tamilnadu, India.

* E-mail: thirusambath5@gmail.com, Tel.: +91-4294-226606, Fax: +91-4294-220087

\begin{abstract}
The key purpose of this study is to explore the ultrasonic assisted extraction (UE) process parameters such as water to sample ratio, ultrasonic time and temperature on the extraction of polysaccharide (PS) from Clitocybe maxima Stipe. Central composite design (CCD) was applied to develop the second order polynomial model. Interactive effects of process variables on the yield of PS was studied using 3D response surface plots. Actual versus predicted plots indicated high-quality association between experimental and predicted values. Water to sample ratio of 50 , ultrasonication time $35 \mathrm{~min}$ of and temperature $45^{\circ} \mathrm{C}$ of gives the maximum yield of PS (40\%). The obtaine crude polysaccharide extract was examined using Fourier transform IR spectrophotometer (FT-IR). The obtained extract is purified through ethanol precipitation and their molecular weight, monosaccharide composition were determined using high performance liquid chromatography (HPLC). The DPPH radical scavenging activity, Hydroxyl radical scavenging assay, Superoxide radical scavenging assay, Reducing power assay, Total antioxidant capacity assay were determined which indicates the effieicency of extracted polysaccharide.
\end{abstract}

Keywords: Polysaccharide, Ultrasonic Extraction, Clitocybe maxima Stipe, Central composite design, High performance liquid chromatography, Scavenging assay.

Paper cited: Thirugnanasambandham, K. (2018). Andioxidant and structural analysis of polysaccharide extracted from Clitocybe maxima Stipe using ultrasonication: simulation and validation. South Asian Journal of Food Technology and Environment, 4(2): 700-708.

\section{Introduction}

Polysaccharides, extensively dispersed in plants, animals, and microorganisms, are interesting resources of additives for drug and food industry, due to their sole chemical and biological activities. In general, botanical polysaccharides are extracted using water or aqueous organic solvents etc ( $\mathrm{He}$ et al., 2014). However, as the cell wall of sample consists of complex polymers, it is not simple to catch out dynamic polysaccharides via extraction process which uses solvent. Thus, some different methods such as microwaving, ultrasonication, enzymatic action, the pressurised water extraction method, etc are also used to improve the polsaccharide extraction process (Contreras et al., 1999). The merits of these techniques are their reduced extraction time, lower solvent consmption and maximum yield of PS.
Response surface methodlogy (RSM) incorporated with CCD is a mixture of statistical and mathematical techniques which is useful for examining the effects of many independent process variables on the process efficiency without the necessitate of a prearranged connection between the objective function and the process variables (ElNawawi et al., 1988). Mean while, the association between the process efficiency value and the independent independent variables is usually unknown in a extraction process; therefore the key step in CCD is to estimated the function (process efficiency) through analyzing process factors. Usually, this process employs a low-order polynomial model in a prearranged region of the extraction process variables. If there is a curve in the process efficiency value, then a polynomial of higher degree, such as a second-order model, have to be used to estimated the process efficiency, 
which is later investigated to place the optimum values of process factors for the maximum process efficiency of any extraction process (Sridhar et al., 2012).

Clitocybe maxima (Gartn. ex Mey. : Fr.) Quél also known as "Da Bei San" or "Zhu Du Gu", is Rare Mushrooms, which developed strongly nowadays (Wang et al., 2007). The advantages Clitocybe maxima are unique flavor, fresh and tender, rich nutrition, and also very favorable to human body health. So, it attracted the very large number of consumers. It characters with a high proportion in stipe bio-mass, which contributes about $45-65 \%$ of entire fruit weight. The stipe is longer than $10 \mathrm{~cm}$, with diameter of $1.5-2.5 \mathrm{~cm}$. Eventhough, the stipe contains slightly minor protein and amino acid than its cap, it yet contains high in fibrous components (Bashi et al., 2012). Hence, the mushroom stipe has been treated as biowaste with very low commercial price still now. The daily yield of the stipe could reach 30-50 tons in a city (Siddiqui et al., 2004). Therefore, there is a critical need to develop the techniques in use of stipe with its characters of high nutrition and fibrous.

Ultrasound-assisted extraction (UE) is an efficient extraction technique that has been used in the extraction of countless kinds of polysaccharides. The auditory cavitation in UE can demolish cell walls, reduce particle sizes and improve the contact between solvents and targeted samples. Moreover, the UE method also has some compensation, such as minor energy consumption, lesser solvent consumption, superior extraction efficiency and a upper level of automation (Xianzhe et al., 2011). The UE extraction conditions such as water to sample ratio, ultrasonic time and temperature could considerably authority the yield and structural characteristic of the polysaccharides, as well as the biological activities (Hu et al., 2014).

According to previous publications, it was noticeable that researches on polysaccharides of Clitocybe maxima Stipe in the last decade was scattered. To improve the supplementary value of bamboo resources, the polysaccharides from various parts of bamboo have been cut off and characterized. However, the extraction of polysaccharides extracted from the Clitocybe maxima Stipe are far from being understood. Thus, in this work, we reported the optimal conditions for the ultrasonic-assisted extraction of novel polysaccharides from the from Clitocybe maxima
Stipe using CCD. Finally, the DPPH radical scavenging activity, Hydroxyl radical scavenging assay, Superoxide radical scavenging assay, Reducing power assay, Total antioxidant capacity assay were determined.

\section{Materials and methods}

Samples: Clitocybe maxima Stipe were harvested and they were air-dried in mature stage at $40{ }^{\circ} \mathrm{C}$ and ground into fine particles through a $0.5 \mathrm{~mm}$ sieve into powder (40 mesh). This sample was stored to analysis in the dark.

Polysaccharide Extraction: The ground dried Clitocybe maxima Stipe samples (5.0 g) were extracted with distilled water, meanwhile the temperature of the water bath was changed. The water-sample slurry was located into a $350 \mathrm{ml}$ conical flask in the ultrasonic bath with ultrasonic time ranging from 2 to $40 \mathrm{~min}$ for a constant power of $150 \mathrm{~W}$ during the process of PS extraction. Finally the extract was centrifuged at $2000 \times g$ for $10 \mathrm{~min}$ to collect the supernatant. The insoluble residue obtained was treated again as mentioned above.

The supernatant was incorporated and concentrated to one-fifth of initial quantity using a rotary evaporator at $55^{\circ} \mathrm{C}$ under vacuum. The resulting solution was assorted with four volumes of dehydrated ethanol (ethanol final concentration, $80 \%$ ) and reserved overnight at $4{ }^{\circ} \mathrm{C}$. Then the solution was centrifuged at $2000 \times g$ for $20 \mathrm{~min}$, washed five times with dehydrated ethanol, and the precipitate was composed. The extract was air-dried at $40^{\circ} \mathrm{C}$ until its weight was steady.

Determination of polysaccharides: Phenolsulfuric acid method (Wang et al., 2007) was used to measure the PS content.. Briefly, in $10 \mathrm{~mL}$ test tubes, $1 \mathrm{~mL}$ of appropriately diluted mixture was

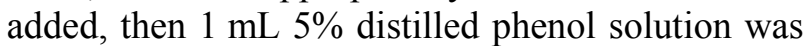
added. The mixture was well shaked after a quick addition of $5 \mathrm{~mL}$ concentrated sulfuric acid. The solution was kept at room temperature for $30 \mathrm{~min}$. The absorbance of the mixture $(150 \mu \mathrm{L})$ was determined at $490 \mathrm{~nm}$. The results were based on a calibration curve obtained using glucose standard solutions. The percentage of polysaccharides yield (PS, \%) is calculated as following equation (Chen et al., 2012)

PS Yield $(\%)=\left(\mathrm{m}_{0} / \mathrm{m}\right) \times 100 \quad--(1)$ 
where, $\mathrm{m}_{0}(\mathrm{~g})$ is the weight of dried polysaccharid and $\mathrm{m}(\mathrm{g})$ is the weight of dried BSS powder.

CCD Experimental design: RSM coupled with (CCD) was employed to explore and optimize the individual and interactive effects of UE process variables such as water to sample ratio, ultrasonic time and temperature on the UE extraction process. Whereas PS production $\left(\mathrm{Y}_{1}\right)$ was selected as response. The CCD results were fixed into the empirical polynomial model, as shown in the following equation (Prakash Maran et al., 2013a) $\mathrm{Y}=\beta_{0}+\sum_{\mathrm{j}=1}^{\mathrm{k}} \beta_{\mathrm{j}} \mathrm{X}_{\mathrm{j}}+\sum_{\mathrm{j}=1}^{\mathrm{k}} \beta_{\mathrm{jj}} \mathrm{X}_{\mathrm{j}}^{2}+\sum_{\mathrm{i}} \sum_{\mathrm{j}=2}^{\mathrm{k}} \beta_{\mathrm{ij}} \mathrm{X}_{\mathrm{i}} \mathrm{X}_{\mathrm{j}}+\mathrm{e}_{\mathrm{i}}---2$

where, $Y$ is the yield of PS; $X_{i}$ and $X_{j}$ are extraction processvariables ( $i$ and $j$ range from 1 to $k$ ); $\beta_{0}$ is the model intercept coefficient; $\beta_{\mathrm{j}}, \beta_{\mathrm{jj}}$ and $\beta_{\mathrm{ij}}$ are interaction coefficients of linear, quadratic and the second-order stipulations, respectively; $k$ is the number of process parameters ( $k=4$ in this study); and $e_{i}$ is the error. Then the sufficiency of the developedmodel was examined using analysis of variance (ANOVA) and actual versus predicted plot. Finally, optimization of UE extraction process variables were prepared according to numerical optimization method. Statistical analyses were done with the use of Design Expert 8.0.7.1 statistical software.

Analytic method: IR spectra of sample was acquired on an FT-IR spectrophotometer. The sample was ground with potassium bromide $(\mathrm{KBr})$ powder and analysed over the wave number range of $4000-400 \mathrm{~cm}^{-1}$. The obtained extract is purified through ethanol precipitation and their molecular weight was determined using high performance liquid chromatography (HPLC). Monosaccharide composition was analyzed by Thin Layer chromatography (TLC).

Evaluation methods of antioxidant activity of TPS: The DPPH radical scavenging activity, Hydroxyl radical scavenging assay, Superoxide radical scavenging assay, Reducing power assay, Total antioxidant capacity assay were determined using statand ard procedure described in elsewhere (Lei et al., 2016).

\section{Results and discussions}

Development and analysis of mathematical model: Table 1 shows the results of the UE process, experimentally calculated responses for the 20 runs according to the CCD experimental design.
The yield of the polysaccharides (PS) ranged from 0.3 to $4 \%$. Based on these data, the UE extraction process was optimized for obtaining desirable response at maximum level. The obtained results of the RSM experiments were analysed with Design Expert software (Yeoh et al., 2008). An ANOVA study for the quadratic mathematical model was used to assess the collision and consequence of each term (linear terms, squared terms and interactions) in the regression model, and results are shown in Table 2. The obtained results of analysis of variance (ANOVA) showed a significant result for the developed mathematical model (Jing et al., 2014). The major involvement of each coefficient was determined by $p$-value of $F$ test $(p<0.05)$. The corresponding process variables become more successful as the $p$-value becomes smaller. Also, the $p$-value can be employed to ensure the interaction strength between independent extraction process factors. Low values of coefficient of variance clearly showed that the developed mathematical model was reproducible and reliable (Mehrotra et al., 2004). A positive sign for the coefficient showed that the tested process variables had a synergistic effect on the polysaccharides yield (PS), while a negative coefficient indicated an antagonistic effect on the response. Results showed that water to sample ratio, ultrasonic time and temperature had a significant positive linear effect on polysaccharides yield. The interaction between $\mathrm{AB}$ and $\mathrm{AC}$ was synergistic and significant. The quality of the fit second order polynomial model can be determined based on the coefficient of determination $\left(R^{2}\right)$ which was at 0.9785 for polysaccharides yield (PS). Significant adequacy of the model was established at the $0.0001 \%$ level of probability with the $R^{2}$ and adjusted- $R^{2}$ of $>88 \%$ (Niu et al., 2013). The obtained equations with coded factors are given below,

$$
\begin{aligned}
& \mathrm{Y}_{1}=4.01+0.61 \mathrm{~A}+0.54 \mathrm{~B}+0.32 \mathrm{C}+0.26 \mathrm{AB}+0.41 \mathrm{AC}- \\
& 0.56 \mathrm{BC}-0.96 \mathrm{~A}^{2}-0.76 \mathrm{~B}^{2}-0.36 \mathrm{C}^{2}---(3)
\end{aligned}
$$

Where, $\mathrm{Y}_{1}$ is yield of PS; A, B, C and D are water to sample ratio, ultrasonic time and temperature, respectively. Meanwhile, the actual and predicted plot of production of PS are plotted in Fig. 1. The obtained data points on this plot lie reasonably close to the straight line. This indicated that an sufficient agreement between real response value and the data obtained from the model. These results showed that the second order model equation developed by RSM could effectively be 
used to portray the UE process under a wide range of extraction process conditions studied.

Table 1: CCD experimental design with results

\begin{tabular}{|c|c|c|c|c|}
\hline S. No. & A & B & C & Y1 \\
\hline 1 & 35.0 & 30.0 & 45.0 & 40 \\
\hline 2 & 10.0 & 40.0 & 35.0 & 20 \\
\hline 3 & 60.0 & 40.0 & 35.0 & 29 \\
\hline 4 & 60.0 & 20.0 & 35.0 & 11 \\
\hline 5 & 10.0 & 40.0 & 55.0 & 30 \\
\hline 6 & 35.0 & 30.0 & 28.2 & 24 \\
\hline 7 & 35.0 & 30.0 & 45.0 & 40 \\
\hline 8 & 35.0 & 30.0 & 45.0 & 40 \\
\hline 9 & 10.0 & 20.0 & 35.0 & 30 \\
\hline 10 & 10.0 & 20.0 & 55.0 & 15 \\
\hline 11 & -7.0 & 30.0 & 45.0 & 0 \\
\hline 12 & 35.0 & 30.0 & 45.0 & 40 \\
\hline 13 & 35.0 & 13.2 & 45.0 & 61 \\
\hline 14 & 60.0 & 40.0 & 55.0 & 35 \\
\hline 15 & 35.0 & 30.0 & 45.0 & 40 \\
\hline 16 & 35.0 & 30.0 & 61.8 & 40 \\
\hline 17 & 35.0 & 30.0 & 45.0 & 40 \\
\hline 18 & 35.0 & 46.8 & 45.0 & 35 \\
\hline 19 & 77.0 & 30.0 & 45.0 & 20 \\
\hline
\end{tabular}

Table 2: ANOVA results

\begin{tabular}{|c|c|c|c|c|c|}
\hline \multicolumn{7}{|c|}{ Y } \\
\hline Source & Sum of Squares & Df & Mean square & F value & P-value \\
\hline Model & 35.36 & 9.0000 & 3.92928 & 12.8638 & 0.0002 \\
\hline A & 5.14 & 1.0000 & 5.14426 & 16.8415 & 0.0021 \\
\hline B & 3.99 & 1.0000 & 3.98504 & 13.0464 & 0.0048 \\
\hline C & 1.41 & 1.0000 & 1.41173 & 4.62176 & 0.0571 \\
\hline AB & 0.55 & 1.0000 & 0.55125 & 1.8047 & 0.2088 \\
\hline AC & 1.36 & 1.0000 & 1.36125 & 4.45651 & 0.0609 \\
\hline BC & 2.53 & 1.0000 & 2.53125 & 8.2869 & 0.0164 \\
\hline A2 & 13.26 & 1.00 & 13.26 & 43.40 & $<0.0001$ \\
\hline B2 & 8.42561 & 1 & 8.42561 & 27.5841 & 0.0004 \\
\hline C2 & 1.84742 & 1 & 1.84742 & 6.04816 & 0.0337 \\
\hline
\end{tabular}

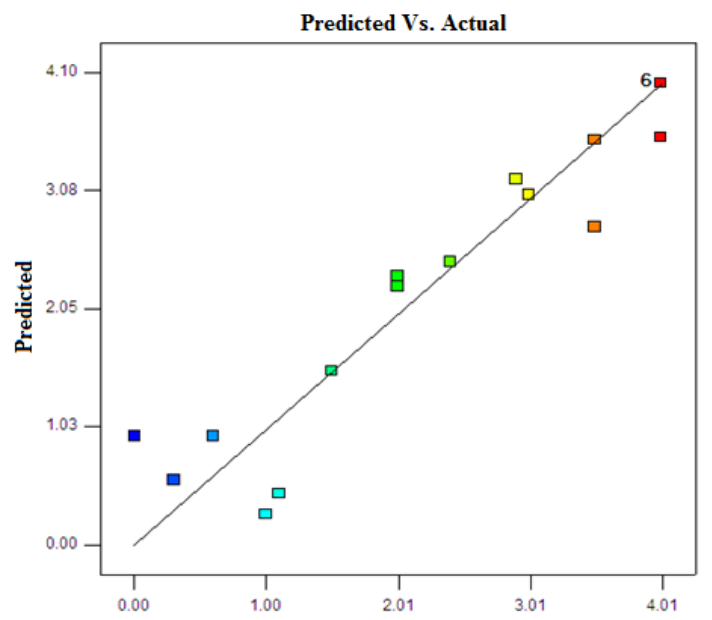

Fig.1: Predicted versus actual plot of production of PS. 

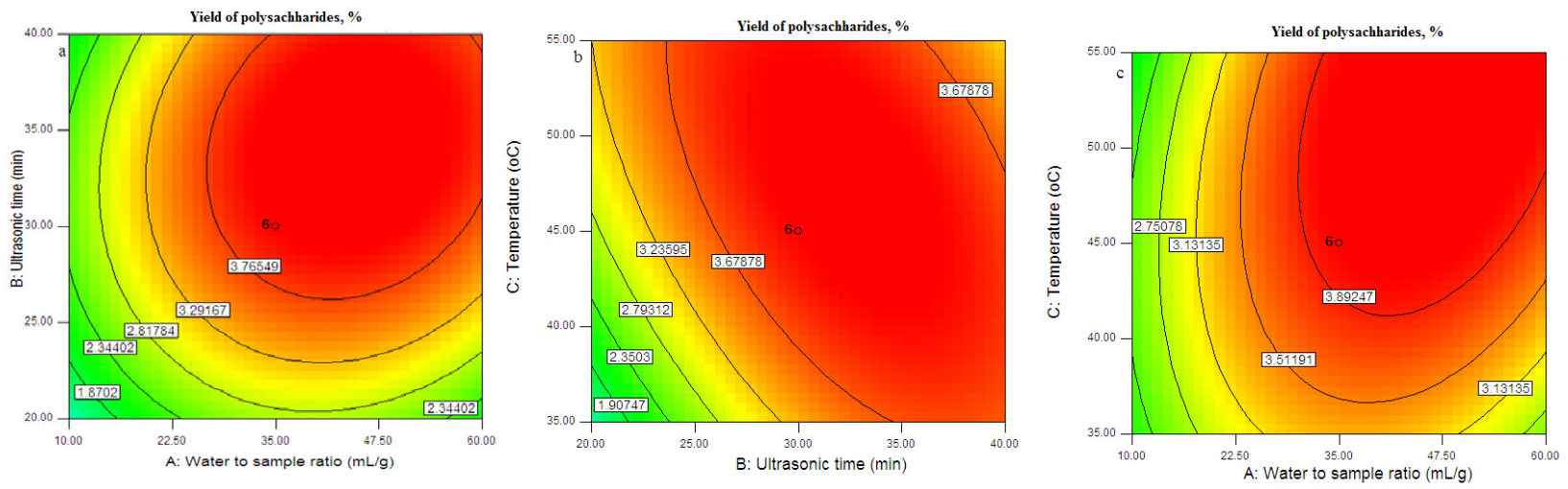

Fig.2: Response surface plots representing the effect of process variables

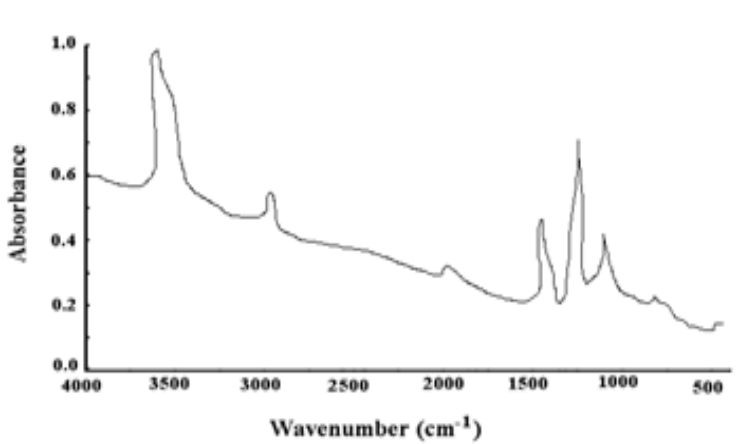

Fig.3: FT-IR spectra of polysaccharide.

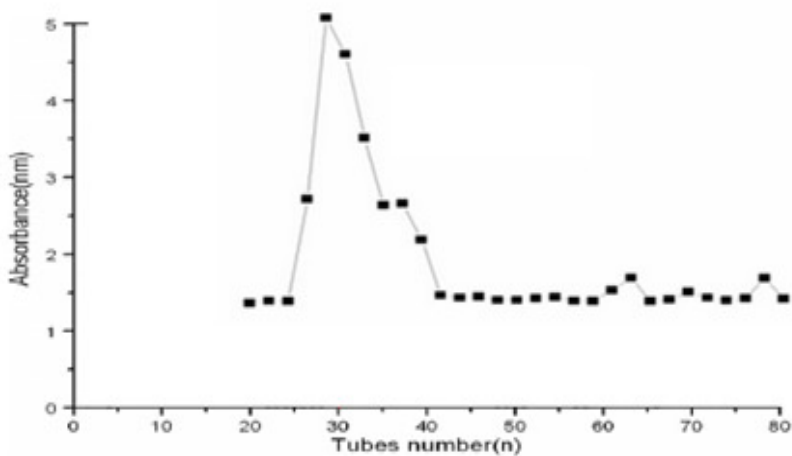

Fig.4: Elution curve of polysaccharide

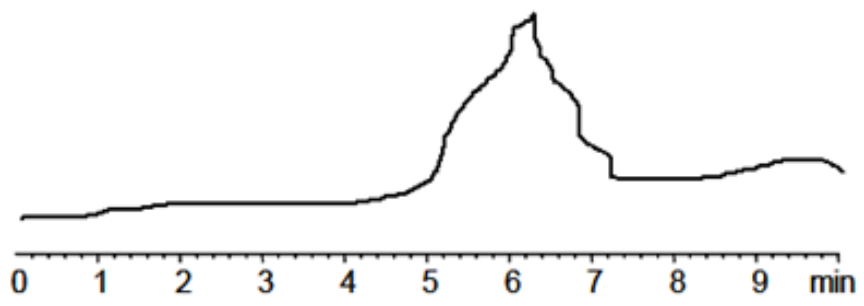

Fig.5: HPLC analysis of polysaccharide

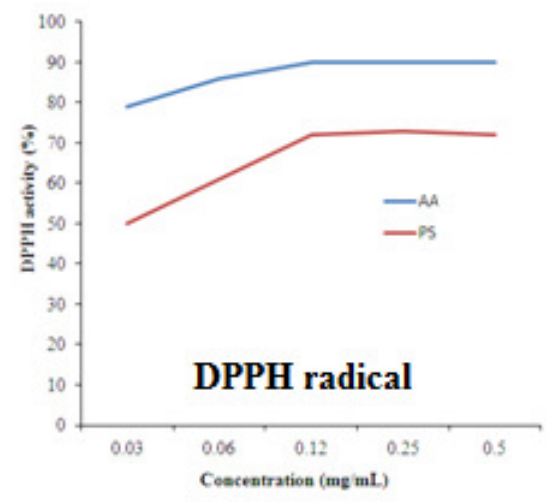

A

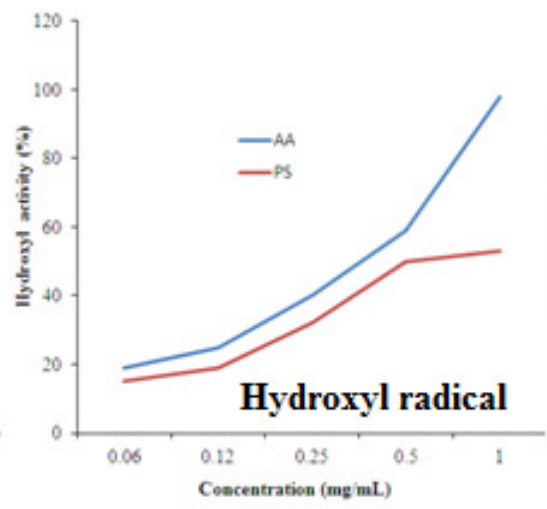

B

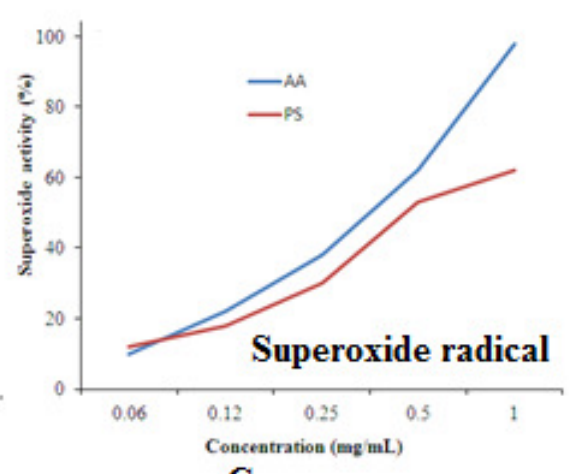

C

Fig. 6: Free radical scavenging activities of ascorbic acid and $\mathrm{PS}$ (mean $\pm \mathrm{SD}, n=3, P<0.01$ ) 
Effect of process conditions on the UE process: From the developed mathematical models, three dimentional plots were designed in order to study the interactive effect of the extraction process variables on the PS yield and it is shown in Fig. 2. In this extraction study, the model has more than two independent process variables. So, the 3D contour plots are drawn by maintaining one process ariable at a constant level, while the other two process variables were varied in their choice.

As shown in Fig. 2A, the polysaccharides yield (PS) increased slowly with increasing water to sample ratio, eventually reaching a highest at 45. These obtained results also indicate that water to sample ratio has a strong positive effect on polysaccharides yield, as it is below 44 . The effect is not significant when water to sample ratio is above 45. As shown in Fig. 2B, the polysaccharides yield (PS) increased slowly with increasing ultrasonic time, in due course reaching a maximum at $35 \mathrm{~min}$ (Park and Jhon, 2009). These consequences also point out that extraction time has a significant positive effect on PS ield when it is below $35 \mathrm{~min}$. The effect is not significant when extraction time is above $35 \mathrm{~min}$. As shown in Fig. $2 \mathrm{C}$, the response value $\left(\mathrm{Y}_{1}\right)$ increased slowly with increasing temperature, in the end reaching a maximum at $40{ }^{\circ} \mathrm{C}$ (Prakash Maran et al., 2013a). These results also point toward that extraction temperature has a considerable positive effect on polysaccharides yield when it is below $40^{\circ} \mathrm{C}$.

Multi response optimization: According to CCD results, optimal UE process conditions to obtain the maximum PS production from Clitocybe maxima Stipe is predicted by Derringer's desired function methodology as follows: Water to sample ratio of 50 , ultrasonic time $35 \mathrm{~min}$ of and temperature $45{ }^{\circ} \mathrm{C}$. Under these variable limits, $40 \%$ of PS are determined with a desirability value of 0.97 . In order to authenticate the optimum process limits, triplicate extraction experiments were done under the optimized conditions and obtained values are close concurrence with predicted values from second order polynomial equation.

Analytic examination of extract: FT-IR analysis was used to find out the functional grops present in the crude extract. The different absorption bands of the FT-IR spectrum (Fig.3) was identified. Strong overlapping IR bands from
1210-1035 $\mathrm{cm}^{-1}$ were attributed to CO stretching vibrations The broadband at $3425 \mathrm{~cm}^{-1}$ indicates $\mathrm{OH}$ stretching vibrations. The band at $1420 \mathrm{~cm}^{-1}$ was due to CO bending vibration. The weak band at $2918 \mathrm{~cm}^{-1}$ was owing to the $\mathrm{CH}$ stretching vibrations of $-\mathrm{CH}^{2}$.There was no absorption near $1700 \mathrm{~cm}^{-1}$ indicating no uronic acid inthe polysaccharide configuration. These outcomes clearly stated that the PS crude extract has polysaccharide functional group in its structure. Then, crude polysaccharides obtained from Clitocybe maxima Stipe were further isolated with DEAE Sepharose Fast Flow ion exchange column and Sephadex G-100 column. The obtained fraction is (Fig.4) named BPS-1. Then the calibration curve was determined by using various standard dextrans. After calculating, the molecular weights of BPS-1 is found to be 589641D (Cho et al., 2003). Based on the retention time of standard monosaccharides, BPS-1 was assumed that composed of rhamnose, arabinose and glucose with a molar ratio of 1:5.25:16.89 (Fig.5).

Antioxidant activity analysis of PS: In order to find out the of antioxidant activities of the PS various experiments were carried out. Fig. 6a shows the scavenging activities for DPPH radical of PS. The shows that the DPPH free radical scavenging rate of PS and positive control ascorbic acid (AA) increased from 52 and 79 to 79 and 91 , when the concentration varied from 0.03125 to $0.5 \mathrm{mg} / \mathrm{mL}$. The results indicated that PS have significant scavenging activity on the DPPH radical, but lower than that of AA $(P<0.01)$.

As the hydroxyl free radical is more likely to be produced in vivo and the most reactive radical in living organisms. Therefore studies were conducted to examine the hydroxyl radical scavenging activity of PS. As shown in Fig. 6b, the hydroxyl radical scavenging activity of PS changed from 15 to $57 \%$, when the concentration ranged from 0.0625 to $1.0 \mathrm{mg} / \mathrm{mL}$. This result suggested that PS have a certain scavenging effect on hydroxyl radical, but with an efficiency far lower than that of AA.

Superoxide anion free radicals can induce the generation of hydrogen peroxide and hydroxyl radical through dismutation and other chemical reactions (Guo et al., 2014). Fig. $6 \mathrm{c}$ indicates that the scavenging effect of PS on 
superoxide radical increased from 14 to 61 , when the concentration increased from 0.0625 to $1.0 \mathrm{mg} / \mathrm{mL}$. But the activity was far lower than that of AA. The results indicated that PS have reasonable scavenging activity over superoxide anion radicals.

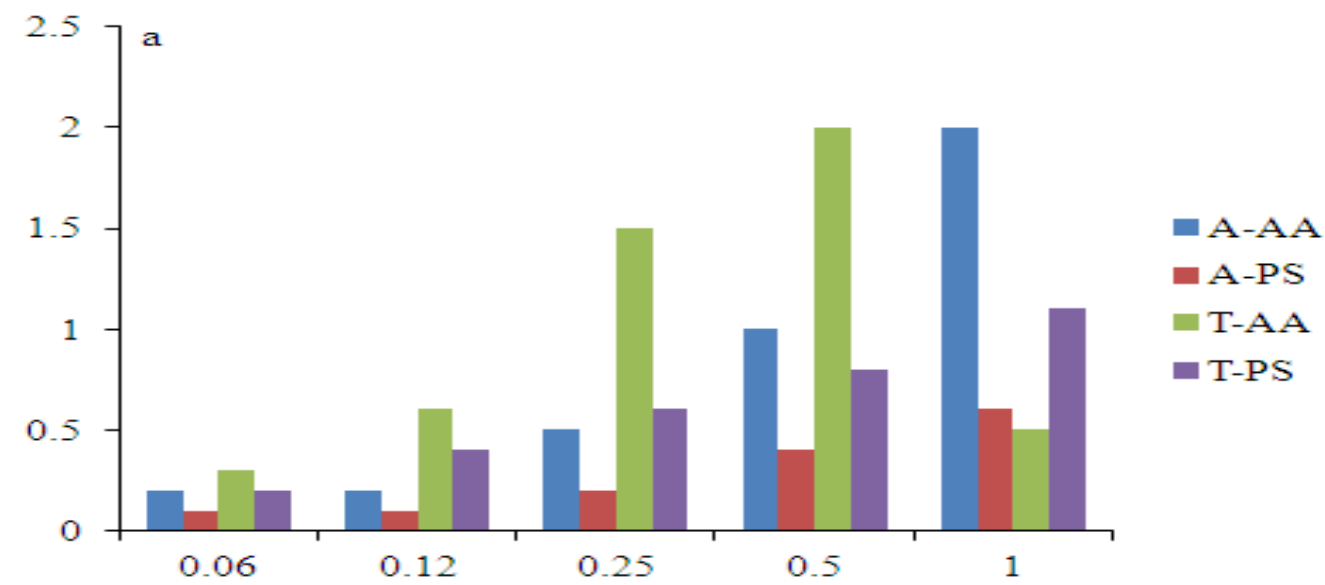

Fig. 7a: Reducing powers of ascorbic acid and PS (mean $\pm \mathrm{SD}, n=3)$.

A-AA and A-PS represents the absorbance at $700 \mathrm{~nm}$ of ascorbic acid and PS respectively; T-AA and T-PS represents the total antioxidant capacity of ascorbic acid and PS respectively.

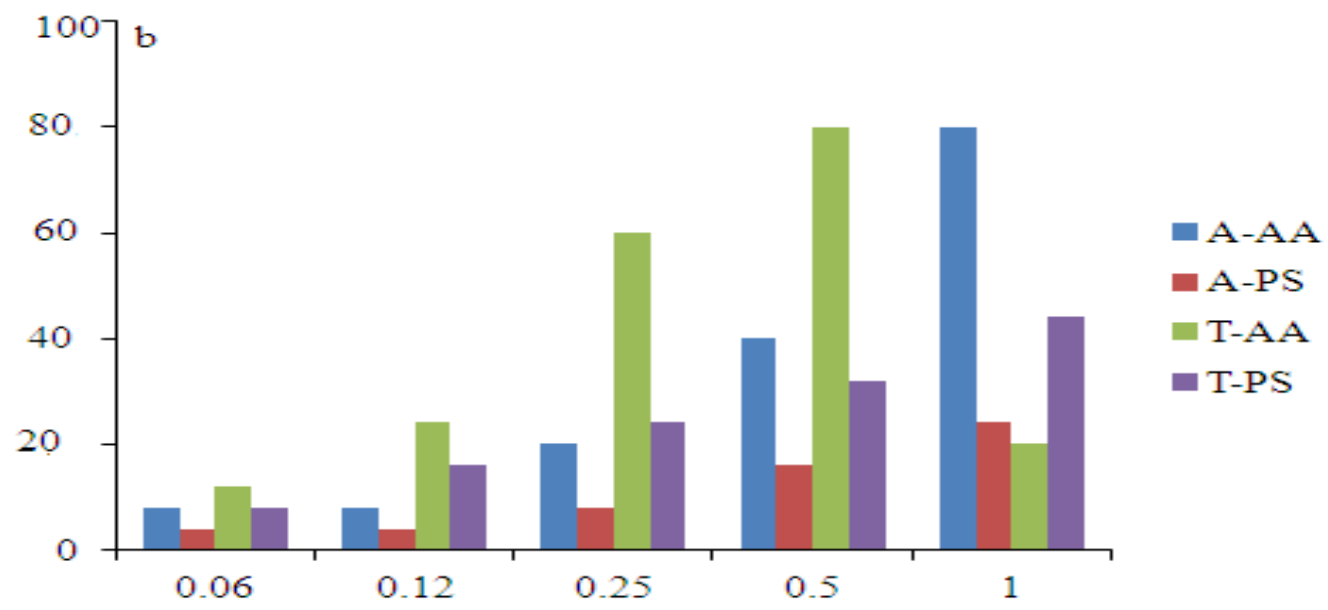

Fig. 7b: Total antioxidant capacities of ascorbic acid and PS (mean $\pm \mathrm{SD}, n=3$ ).

A-AA and A-PS represents the absorbance at $700 \mathrm{~nm}$ of ascorbic acid and PS respectively;

T-AA and T-PS represents the total antioxidant capacity of ascorbic acid and PS respectively.

In this study, the very closely correlation between antioxidant capacity and reducing power were studied. The antioxidant could reduce $\mathrm{Fe}^{3+} /$ ferricyanide complex to the $\mathrm{Fe}^{2+}$, in the reducing power test. For now, the $\mathrm{Fe}^{2+}$ can be measured by monitoring the formation of Perl's Prussian blue at $700 \mathrm{~nm}$ (Yuan et al., 2015). Hence, the absorbance were calculated against concentration and the results were shown in Fig. 7a and $7 \mathrm{~b}$. The stronger absorbance of the mixture confirmed the greater reducing power. Fig. 7 showed the reducing power and total antioxidant capability of PS increases with concentration, but the efficiency was lower than that of AA.

\section{Conclusion}

In this present reserch work, production of polysaccharide from Clitocybe maxima Stipe is examined under process parameters such as water to sample ratio, ultrasonic time and temperature using Ultrasonic assisted extration (UE). RSM coupled CCD with four variable design was used to develop the second order polynomial model. Analysis of variance was used to study the capability of developed second order polynomial 
equation. Water to sample ratio of 50 , ultrasonic time $35 \mathrm{~min}$ of and temperature $45^{\circ} \mathrm{C}$ of gives the maximum yield of PS (40\%). The obtaine crude polysaccharide extract was examined using Fourier transform IR spectrophotometer (FT-IR) and results exhibited the presence of polysaccharide functional groups. The obtained extract is purified through ethanol precipitation and their molecular weight was determined using high performance liquid chromatography (HPLC). The DPPH radical scavenging activity, Hydroxyl radical scavenging assay, Superoxide radical scavenging assay, Reducing power assay, Total antioxidant capacity assay were determined.

\section{References}

1. Bashi, D.S., Mortazavi, S.A., Rezaei, K., Rajaei, A., Karimkhani, M.M., (2012). Optimization of ultrasound-assisted extraction of phenolic compounds from Yarrow (Achillea beibrestinii) by response surface methodology. Food Science and Biotechnology, 21 (4): 1005-1011.

2. Chen, Y., Xie, M., Li, W., Zhang, H., Nie, S., Wang, Y., (2012). An effectivemethod for deproteinization of bioactive polysaccharides extracted fromlingzhi (Ganoderma atrum). Food Science and Biotechnology, 21: 191-198.

3. Cho, C.W., Lee, D.Y. and Kim, C.W. (2003). Concentration and purification of soluble pectin from mandarin peels using cross flow micro-filtration system. Carbohydrate Polymers, 54(1): 21-26.

4. Contreras-Esquivel, J.C., Hours, R.A., Voget, C.E., \& Mignone, C.F. (1999). Aspergillus kawachii - Produces an acidic pectin releasing enzyme activity. Journal of Bioscience and Bioengineering, 88(1): 4852.

5. El-Nawawi, S.A., and Shehata, F. R. F. R. (1988). Effect of the extraction temperature on the characteristics of pectin extracted from Egyptian orange peel. Biological Wastes, 24: 307-311.

6. Guo, L., Zhu, W., Xu, F., Liu, M., Xie, Y., Zhang, J. (2014). Optimized ultrasonicassisted extraction of polysaccharides from Cyclina sinensis and evaluation of antioxidant activities in vitro. CyTA-Journal of Food, 12: 32-39.
7. He, M. X., Wang, J. L., Qin, H., Shui, Z. X., Zhu, Q. L., Wu, B. (2014). Bamboo: anew source of carbohydrate for biorefinery. Carbohydrate Polymers, 111: 645-654.

8. Hu, J. L., Nie, S. P., Wu, Q. M., Li, C., Fu, Z. H., Gong, J. (2014). Polysaccharidefrom seeds of Plantago asiatica L. affects lipid metabolism and colon microbiotaof mouse. Journal of Agricultural and Food Chemistry, 62: 229-234.

9. Jing, Y., Huang, L., Lv, W., Tong, H., Song, L., Hu, X., et al. (2014). Structuralcharacterization of a novel polysaccharide from pulp tissues of Litchi chinensisand its immunomodulatory activity. Journal of Agricultural and Food Chemistry, 62: 902-911.

10. Lei, Guo., Jiacai, Guo., Wencheng, Zhu., \& Xinru, Jiang. (2016). Optimized synchronous extraction process of tea polyphenols and polysaccharides from Huaguoshan Yunwu tea and their antioxidant activities. Food and Bioproducts Processing, 100: 303-310.

11. Mehrotra, R., Bajaj, S., and Kumar, D. (2004). Use of complementary and alternativemedicine by patients with diabetes mellitus. National Medical Journal of India, 17: 243-245.

12. Niu, Y., Yan, W., Lv, J., Yao, W., \& Yu, L. L. (2013). Characterization of a novelpolysaccharide from tetraploid Gynostemma pentaphyllum makino. Journal of Agricultural and Food Chemistry, 61: 4882-4889.

13. Park, E. J., and Jhon, D. Y. (2009). Effects of bamboo shoot consumption on lipidprofiles and bowel function in healthy young women. Nutrition, 25: 723-728.

14. Prakash Maran, J., Sivakumar, V., Sridhar, R., and Prince Immanuel, V. (2013a). Development of model for mechnical properties of tapioca starch based edible films. Industrial Crops and Products, 42: 159-168.

15. Prakash Maran, J., Sivakumar, V., Thirugnanasambandham, K., and Sridhar, R. (2013b). Model development and process optimization for solvent extraction of polyphenols from red grapes using BoxBehnken design. Preparative Biochemistry 
and

Biotechnology.

DOI:10.1080/10826068.2013.791629.

16. Siddiqui, I.A., Afaq, F., Adhami, V.M., Ahmad, N., Mukhtar, H. (2004). Antioxidants of the beverage tea in promotion of human health. Antioxidants and Redox Signaling, 6: 571-582.

17. Sridhar, R., Sivakumar, V., Prince Immanuel, V., and Prakash Maran, J. (2012). Development of model for treatment of Pulp and Paper industry bleaching effluent using response surface methodology. Environmental Progress \& Sustainable Energy, 31: 558-565.

18. Wang, S.J., Chen, F., Wu, J.H., Wang, Z.F., Liao, X.J., and Hu, X.S. (2007). Optimization of pectin extraction assisted by microwave from apple pomace using response surface methodology. Journal of Food Engineering, 78(2): 693-700.

19. Xianzhe, Z., Fangping, Y., Chenghai, L., and Xiangwen, X. (2011). Effect of process parameters of microwave assisted extraction (MAE) on polysaccharides yield from Pumpkin. Journal of Northeast Agricultural University, 8(2): 79-86.

20. Yeoh, S., Shi, J., and Langrish, T. A. G. (2008). Comparisons between different techniques for water-based extraction of pectin from orange peels. Desalination, 218: 229-237.

21. Yuan, C., Li, Z., Peng, F., Xiao, F., Ren, D., Xue, H., Chen, T., Mushtaq, G. and Kamal, M.A. (2015). Combination of seleniumenriched green tea polysaccharides and Huoji polysaccharides synergistically enhances antioxidant and immune activity in mice. Journal ofthe Science of Food and Agriculture, $95: 3211-3217$.

$\begin{array}{ll}\text { Received } & \text { : Aug., } 2018 \\ \text { Revised } & \text { : Oct., } 2018 \\ \text { Published } & \text { : Dec., } 2018\end{array}$

